\title{
Application of a modified Adomian decomposition method to solving a kind of wave equation
}

\author{
Francis Bassono ${ }^{1}$, Pare Youssouf ${ }^{1 *}$, Bissanga Gabriel ${ }^{2}$, Some Blaise ${ }^{1}$ \\ ${ }^{1}$ University of Ouagadougou \\ ${ }^{2}$ University Marien Ngouabi of Brazzaville \\ *Corresponding author E-mail: pareyoussouf@yahoo.fr
}

\begin{abstract}
In this paper, a modified Adomian decomposition method is used to construct the solution of the initial value problem of a wave equation.
\end{abstract}

Keywords: Modified Adomian decomposition method, wave equation.

\section{Introduction}

The wave equation is used in many areas among them: electronic, physics, mechanics. The Adomian decomposition method (ADM) is very useful to get an approximation of the solution of an equation. In the last years, the ADM has been applied to get the solutions of various kinds of problems of EDEs and PDEs [1]-[10]. Here, we use a modified Adomian decomposition method proposed in [11], to investigate a wave equation. Using this last method, we get the exact solution.

\section{About modified Adomian decomposition method}

In [11], a new modified Adomian decomposition method is proposed for solving a kind of the evolution equation. Here, we apply this method to wave equation.

\subsection{The standard Adomian decomposition method}

Let's consider the following initial value problem of a wave equation:

$$
\begin{aligned}
& \frac{\partial^{2} u}{\partial t^{2}}=a \frac{\partial u}{\partial t}+\sum_{m=0}^{+\infty} b_{m} \frac{\partial^{m} u}{\partial x^{m}}+\sum_{m=0}^{N} c_{m} \frac{\partial^{m} u^{k+1}}{\partial x^{m}}+d \frac{\partial^{i+1}}{\partial x^{i} \partial t}+f(x, t),-\infty<x<+\infty, t>0 \\
& u(x, 0)=h(x) ; \frac{\partial u(x, 0)}{\partial t}=g(x)
\end{aligned}
$$

where $a, b_{m}, c_{m}$ and $d$ are real constants, $k$ and $i$ are positive integers, $M$ and $N$ are nonnegative integers, $f(x, t)$ is given function and $u(x, t)$ the unknown.

We denote by:

$$
L_{t t} u=\frac{\partial^{2} u}{\partial t^{2}}, R u=a \frac{\partial u}{\partial t}+\sum_{m=0}^{M} b_{m} \frac{\partial^{m} u}{\partial x^{m}}+d \frac{\partial^{i+1}}{\partial x^{i} \partial t}, N u=\sum_{m=0}^{N} c_{m} \frac{\partial^{m} u^{k+1}}{\partial x^{m}}
$$


and $L_{t t}^{-1}=\int_{0}^{t} \int_{0}^{s} d z d s$. Thus we can rewrite (2.1) as

$L_{t t} u=R u+N u+f(x, t)$

From (2.4), we have

$u=h(x)+\operatorname{tg}(x)+L_{t t}^{-1} R u+L_{t t}^{-1} N u+L_{t t}^{-1} f(x, t)$

According to the standard Adomian decomposition method, we suppose that the solution of (2.1) has the following form:

$u(x)=\sum_{n=0}^{+\infty} u_{n}(x)$

and

$N(u(x))=\sum_{n=0}^{+\infty} A_{n}(x)$

where $A_{n}$ are special polynomials of variables $u_{0}, u_{1}, \ldots, u_{n}$ called Adomian polynomials and defined by [4],[5], [6]:

$A_{n}=\frac{1}{n !} \frac{d^{n}}{d \lambda^{n}}\left[N\left(\sum_{i=0}^{+\infty} \lambda^{i} u_{i}\right)\right]_{\lambda=0} ; n=0,1,2, \ldots$

From (2.5); (2.6) and (2.7) we have the following Adomian algorithm:

$\left\{\begin{array}{l}u_{0}=h(x)+\operatorname{tg}(x)+L_{t t}^{-1} f(x, t) \\ u_{n+1}=L_{t t}^{-1} R u_{n}+L_{t t}^{-1} A_{n} ; \forall n \geq 0\end{array}\right.$

\subsection{The modified Adomian decomposition method}

According to the modified Adomian decomposition method [11], the first term $u_{0}$ in (2.6), is to be determined as follows:

$$
u_{0}=h(x)+\operatorname{tg}(x)+L_{t t}^{-1} f(x, t)+\alpha L_{t t}^{-1} G\left(u_{0}\right)
$$

\section{Application}

\subsection{The modified Adomian decomposition method}

Let's use the modified Adomian decomposition method (2.11) and (2.12) to solve the following problem

$$
\left\{\begin{array}{l}
\frac{\partial^{2} u}{\partial t^{2}}+\frac{\partial u}{\partial t}+\frac{\partial^{2} u}{\partial x^{2}}-2 u^{3}+u^{2}=0 \\
u(x, 0)=\frac{1}{x} \\
\frac{\partial u(x, 0)}{\partial t}=-\frac{1}{x^{2}}
\end{array}\right.
$$

we denote 


$$
\begin{aligned}
& L_{t t} u=\frac{\partial^{2} u}{\partial t^{2}}, L_{t} u=\frac{\partial u}{\partial t}, L_{x x} u=\frac{\partial^{2} u}{\partial x^{2}} \\
& R u=-\frac{\partial u}{\partial t}-\frac{\partial^{2} u}{\partial x^{2}}=-L_{t} u-L_{x x} u, N u=4 u^{3}-u^{2}
\end{aligned}
$$

From (3.1), we have

$L_{t t} u=R u+N u$

(3.3) gives us

$$
u=u(x, 0)+\frac{\partial u(x, 0)}{\partial t} t+L_{t t}^{-1} R u+L_{t t}^{-1} N u
$$

From (2.10), (2.11), (2.12) and (2.13) we have following modified Adomian algorithm

$$
\left\{\begin{array}{l}
u_{0}=\frac{1}{x}-\frac{t}{x^{2}}+L_{t t}^{-1} L_{x x} u_{0} \\
u_{1}=L_{t t}^{-1} R u_{0}+L_{t t}^{-1} A_{0}-L_{t t}^{-1} L_{x x} u_{0} \\
u_{n+1}=L_{t t}^{-1} R u_{n}+L_{t t}^{-1} L_{x x} u_{n} ; \forall n \geq 1
\end{array}\right.
$$

Where $u_{0}$ is the solution of the following system:

$$
\left\{\begin{array}{l}
\frac{\partial^{2} v}{\partial t^{2}}=\frac{\partial^{2} v}{\partial x^{2}} \\
v(x, 0)=\frac{1}{x} \\
\frac{\partial v(x, 0)}{\partial t}=-\frac{1}{x^{2}}
\end{array}\right.
$$

To solve (3.6), we use the standard Adomian algorithm (2.9). From (3.6), we have

$v(x, t)=v(x, 0)+\frac{\partial v(x, 0)}{\partial t} t+L_{t t}^{-1} L_{x x} v(x, t)$

We suppose that the solution of (3.6) has the following form

$v(x, t)=\sum_{n=0}^{+\infty} v_{n}(x, t)$

Thus we have the following Adomian algorithm

$$
\left\{\begin{array}{l}
v_{0}(x, t)=v(x, 0)+\frac{\partial v(x, 0)}{\partial t} t \\
v_{n+1}(x, t)=L_{t t}^{-1} L_{x x} v_{n}(x, t) ; n \geq 1
\end{array}\right.
$$

This gives as 
$\left\{\begin{array}{l}v_{0}(x, t)=\frac{1}{x}-\frac{1}{x^{2}} \\ v_{1}(x, t)=\frac{t^{2}}{x^{3}}-\frac{t^{3}}{x^{4}} \\ v_{2}(x, t)=\frac{t^{4}}{x^{5}}-\frac{t^{5}}{x^{6}} \\ v_{3}(x, t)=\frac{t^{6}}{x^{7}}-\frac{t^{7}}{x^{8}} \\ \ldots\end{array}\right.$

\section{Therefore}

$$
\left\{\begin{aligned}
u_{0}(x, t) & =v(x, t)=\frac{1}{x}-\frac{t}{x^{2}}+\frac{t^{2}}{x^{3}}-\frac{t^{3}}{x^{4}}+\frac{t^{4}}{x^{5}}-\frac{t^{5}}{x^{6}}+\frac{t^{6}}{x^{7}}-\frac{t^{7}}{x^{8}}+\ldots \\
& =\frac{1}{x}\left(1-\frac{t}{x}+\frac{t^{2}}{x^{2}}-\frac{t^{3}}{x^{3}}+\frac{t^{4}}{x^{4}}-\frac{t^{5}}{x^{5}}+\ldots\right) \\
& =\frac{1}{x} \sum_{n=0}^{+\infty}\left(\frac{t}{x}\right)^{n} \\
& =\frac{1}{x+t}
\end{aligned}\right.
$$

with $\left|\frac{t}{x}\right|<1$

From (2.8) and (3.5), we have

$$
\begin{aligned}
& A_{0}=N\left(u_{0}\right)=4\left(\frac{1}{x+t}\right)^{3}-\left(\frac{1}{x+t}\right)^{2} \\
& u_{1}=L_{t t}^{-1}\left(R u_{0}+A_{0}-L_{x x} u_{0}\right) \\
& =L_{t t}^{-1}\left[\frac{\partial\left(\frac{1}{x+t}\right)}{\partial t}-\frac{\partial^{2}\left(\frac{1}{x+t}\right)}{\partial x^{2}}+4\left(\frac{1}{x+t}\right)^{3}-\left(\frac{1}{x+t}\right)^{2}-\frac{\partial^{2}\left(\frac{1}{x+t}\right)}{\partial x^{2}}\right] \\
& =L_{t t}^{-1}\left[\left(\frac{1}{x+t}\right)^{2}-2\left(\frac{1}{x+t}\right)^{3}+4\left(\frac{1}{x+t}\right)^{3}-\left(\frac{1}{x+t}\right)^{2}-2\left(\frac{1}{x+t}\right)^{3}\right]=0 \\
& A_{1}=12 u_{0}^{2} u_{1}-2 u_{0} u_{1}=0 \\
& u_{2}(x, t)=L_{t t}^{-1}\left(R u_{1}+A_{1}\right)=0 \\
& A_{2}=12 u_{0} u_{1}^{2}+6 u_{0}^{2}-u_{1}^{2}-u_{0} u_{2}=0 \\
& u_{3}(x, t)=L_{t t}^{-1}\left(R u_{2}+A_{0}\right)=0
\end{aligned}
$$

We can easily get: $A_{n}=0$ and $u_{n}=0$ for $n \geq 1$.

Thus the solution of (3.1) is:

$$
u(x, t)=\sum_{n=0}^{+\infty} u_{n}(x, t)=u_{0}(x, t)=\frac{1}{x+t}
$$




\subsection{The standard Adomian decomposition method}

From (3.1) we have

$$
\frac{\partial u(x, t)}{\partial t}-\frac{\partial u(x, 0)}{\partial t}+u(x, t)-u(x, 0)+\int_{0}^{t} \frac{\partial^{2} u(x, s)}{\partial x^{2}} d s+\int_{0}^{t} N(u(x, s)) d s=0
$$

From (3.13), we have

$$
u(x, t)-u(x, 0)-\frac{\partial u(x, 0)}{\partial t} t+\int_{0}^{t} u(x, s) d s-u(x, 0) t+\int_{0}^{t} \int_{0}^{z} \frac{\partial^{2} u(x, s)}{\partial x^{2}} d s d z+\int_{0}^{t} \int_{0}^{z} N(u(x, s)) d s d z=0
$$

From (2.9) and (3.14), we have the following standard Adomian algorithm

$$
\left\{\begin{array}{l}
u_{0}(x, t)=\frac{1}{x}-\frac{t}{x^{2}}+\frac{t}{x} \\
u_{n+1}(x, t)=-\int_{0}^{t} u_{n}(x, s) d s-\int_{0}^{t} \int_{0}^{z} \frac{\partial^{2} u(x, s)}{\partial x^{2}} d s d z-\int_{0}^{t} \int_{0}^{z} A_{n}(x, s) d s d z ; n \geq 0
\end{array}\right.
$$

which gives us

$$
\begin{aligned}
& u_{1}=\left(\frac{1}{5 x^{3}}-\frac{3}{5 x^{4}}+\frac{3}{5 x^{5}}-\frac{1}{5 x^{6}}\right) t^{5}+\left(\frac{7}{6 x^{3}}-\frac{1}{12 x^{2}}-\frac{25}{12 x^{4}}+\frac{1}{x^{5}}\right) t^{4}+\left(\frac{2}{x^{3}}-\frac{1}{3 x^{2}}-\frac{1}{x^{4}}\right) t^{3}+\left(\frac{1}{x^{3}}-\frac{1}{2 x}\right) t^{2}+\left(-\frac{1}{x}\right) t \\
& u_{2}=\left(\frac{1}{30 x^{5}}-\frac{1}{6 x^{6}}+\frac{1}{3 x^{7}}-\frac{1}{3 x^{8}}+\frac{1}{6 x^{9}}-\frac{1}{30 x^{10}}\right) t^{9}+\left(\frac{2}{5 x^{5}}-\frac{1}{40 x^{4}}-\frac{27}{20 x^{6}}+\frac{19}{10 x^{7}}-\frac{49}{40 x^{8}}+\frac{3}{10 x^{9}}\right) t^{8}+ \\
& \left(\frac{1}{252 x^{3}}-\frac{89}{420 x^{4}}+\frac{697}{420 x^{5}}-\frac{4337}{1260 x^{6}}+\frac{284}{105 x^{7}}-\frac{5}{7 x^{2}}\right) t^{7}+\left(\frac{83}{36 x^{2}}-\frac{1}{60 x^{4}}-\frac{37}{180 x^{3}}-\frac{247}{90 x^{6}}+\frac{3}{5 x^{7}}\right) t^{6}+ \\
& \left(\frac{1}{3 x^{2}}-\frac{37}{12 x^{3}}+\frac{25}{12 x^{4}}\right) t^{5}+\left(\frac{1}{6 x}+\frac{1}{3 x^{2}}-\frac{2}{x^{3}}\right) t^{4}+\frac{1}{2 x} t^{2} \\
& u_{3}=\left(\frac{17}{3900 x^{7}}-\frac{119}{3900 x^{8}}+\frac{119}{1300 x^{9}}-\frac{119}{780 x^{10}}+\frac{119}{780 x^{11}}-\frac{119}{1300 x^{12}}+\frac{119}{3900 x^{13}}-\frac{17}{3900 x^{14}}\right) t^{13}+ \\
& \left(\frac{17}{200 x^{7}}-\frac{17}{3600 x^{6}}-\frac{493}{1200 x^{8}}+\frac{17}{18 x^{9}}-\frac{289}{240 x^{10}}+\frac{527}{600 x^{11}}-\frac{1241}{3600 x^{12}}+\frac{17}{300 x^{13}}\right) t^{12}+ \\
& \left(\frac{139}{92400 x^{5}}-\frac{1977}{30800 x^{6}}+\frac{849}{1400 x^{7}}-\frac{95083}{46200 x^{8}}+\frac{12535}{3696 x^{9}}-\frac{91141}{30800 x^{10}}+\frac{4351}{3600 x^{11}}-\frac{1366}{5775 x^{12}}\right) t^{11}+ \\
& \left(\frac{94901}{56700 x^{7}}-\frac{1997}{15120 x^{6}}+\frac{6469}{226800 x^{5}}-\frac{11}{90720 x^{4}}+\frac{1972183}{453600 x^{8}}-\frac{355927}{75600 x^{9}}+\frac{84487}{37800 x^{10}}-\frac{38}{105 x^{11}}\right) t^{10}+ \\
& \left(\frac{613}{22680 x^{4}}-\frac{43919}{90720 x^{5}}+\frac{85003}{90720 x^{6}}-\frac{9479}{9072 x^{7}}-\frac{152587}{45380 x^{8}}+\frac{8453}{3780 x^{9}}-\frac{173}{420 x^{10}}\right) t^{9}+ \\
& \left(\frac{2603}{10080 x^{4}}-\frac{1}{315 x^{3}}-\frac{1579}{672 x^{5}}+\frac{42577}{10080 x^{6}}-\frac{8357}{5040 x^{7}}-\frac{461}{560 x^{8}}+\frac{99}{140 x^{9}}\right) t^{8}+
\end{aligned}
$$




$$
\begin{aligned}
& \left(\frac{13}{126 x^{3}}+\frac{81}{140 x^{4}}-\frac{1651}{360 x^{5}}+\frac{12991}{2520 x^{6}}-\frac{67}{70 x^{7}}-\frac{6}{35 x^{8}}\right) t^{7} \\
& \left(\frac{313}{360 x^{3}}-\frac{1}{40 x^{2}}-\frac{2}{9 x^{4}}-\frac{239}{72 x^{5}}+\frac{55}{36 x^{6}}+\frac{2}{5 x^{7}}\right) t^{6}+\left(\frac{259}{120 x^{3}}-\frac{3}{20 x^{2}}-\frac{67}{60 x^{4}}-\frac{3}{5 x^{5}}\right) t^{5}+ \\
& \left(\frac{5}{3 x^{3}}-\frac{5}{24 x^{2}}-\frac{1}{24 x}\right) t^{4}+\left(-\frac{1}{6 x}\right) t^{3} \\
& u_{4}=\left(\frac{397}{442000 x^{9}}-\frac{3573}{442000 x^{10}}+\frac{3573}{110500 x^{11}}-\frac{8337}{110500 x^{12}}+\frac{25011}{221000 x^{13}}-\frac{25011}{221000 x^{14}}\right) t^{17}+ \\
& \left(\frac{8337}{110500 x^{15}}-\frac{3573}{11500 x^{16}}+\frac{3573}{442000 x^{17}}-\frac{397}{442000 x^{18}}\right) t^{17}+ \\
& \left(\frac{397}{15600 x^{9}}-\frac{397}{312000 x^{8}}-\frac{12307}{78000 x^{10}}+\frac{19453}{39000 x^{11}}-\frac{147287}{156000 x^{12}}+\frac{5558}{4875 x^{13}}\right) t^{16}+ \\
& \left(-\frac{2779}{3120 x^{14}}+\frac{17071}{39000 x^{15}}-\frac{38509}{312000 x^{16}}+\frac{397}{26000 x^{17}}\right) t^{16}+ \\
& \left(\frac{661}{1078000 x^{7}}-\frac{29611}{1201200 x^{8}}+\frac{1028273}{382200 x^{9}}-\frac{1563901}{1274000 x^{10}}+\frac{126828329}{42042000 x^{11}}-\frac{186596959}{42042000 x^{12}}\right) t^{15}+ \\
& \left(\frac{6810649}{1681680 x^{13}}-\frac{31603181}{14014000 x^{14}}+\frac{1233377}{1751750 x^{15}}-\frac{995191}{10510500 x^{16}}\right) t^{15}+ \\
& \left(\frac{921551}{764400 x^{9}}-\frac{349}{112112 x^{7}}-\frac{31819}{2866550 x^{8}}-\frac{2837}{25225200 x^{6}}-\frac{38124529}{8408400 x^{10}}+\frac{73032979}{8408400 x^{11}}\right) t^{14}+ \\
& \left(-\frac{23824531}{2522520 x^{12}}+\frac{16534753}{2802800 x^{13}}-\frac{24815149}{12612600 x^{14}}+\frac{567311}{2102100 x^{15}}\right) t^{14}+ \\
& \left(\frac{41}{7076160 x^{5}}+\frac{4116053}{389188800 x^{6}}-\frac{35900477}{194594400 x^{7}}+\frac{69251453}{194594400 x^{8}}+\frac{135703553}{77837760 x^{9}}-\frac{2966586941}{389188800 x^{10}}\right) t^{13}+ \\
& \left(\frac{767076589}{64864800 x^{11}}-\frac{193484789}{21621600 x^{12}}+\frac{8823523}{2702700 x^{13}}-\frac{133543}{300300 x^{14}}\right) t^{13}+ \\
& \left(\frac{272743}{1663200 x^{6}}-\frac{11747}{3742200 x^{5}}-\frac{8096521}{4989600 x^{7}}+\frac{43899193}{9979200 x^{8}}-\frac{44782379}{14968800 x^{9}}\right) t^{12}+ \\
& \left(-\frac{23354071}{5987520 x^{10}}+\frac{35038397}{4989600 x^{11}}-\frac{592574}{155925 x^{12}}+\frac{16987}{23100 x^{13}}\right) t^{12}+ \\
& \left(\frac{1027}{4989600 x^{4}}+\frac{1579}{90720 x^{5}}+\frac{721307}{997920 x^{6}}-\frac{7495991}{1247400 x^{7}}+\frac{68582693}{4989600 x^{8}}\right) t^{11}+ \\
& \left(-\frac{136787}{118820 x^{9}}+\frac{1418161}{831600 x^{10}}+\frac{38191}{17325 x^{11}}-\frac{3923}{4620 x^{12}}\right) t^{11}+ \\
& \left(\frac{95233}{181440 x^{5}}-\frac{5773}{226800 x^{4}}+\frac{172993}{302400 x^{6}}-\frac{4641457}{453600 x^{7}}+\frac{149713}{8100 x^{8}}-\frac{2401079}{226800 x^{9}}+\frac{289}{378 x^{10}}+\frac{713}{2100 x^{11}}\right) t^{10}+ \\
& \left(\frac{13}{6480 x^{3}}-\frac{22219}{90720 x^{4}}+\frac{247763}{90720 x^{5}}-\frac{124807}{45360 x^{6}}-\frac{683647}{90720 x^{7}}+\frac{976351}{90720 x^{8}}-\frac{2843}{945 x^{9}}-\frac{89}{210 x^{10}}\right) t^{9}+ \\
& \left(\frac{9407}{1440 x^{5}}-\frac{173}{210 x^{4}}-\frac{179}{5040 x^{3}}-\frac{3131}{480 x^{6}}-\frac{24889}{10080 x^{7}}+\frac{4709}{1689 x^{8}}-\frac{17}{70 x^{9}}\right) t^{8}+
\end{aligned}
$$




$$
\begin{aligned}
& \left(\frac{1}{105 x^{2}}-\frac{85}{168 x^{3}}-\frac{271}{315 x^{4}}+\frac{2597}{360 x^{5}}-\frac{4831}{1260 x^{6}}-\frac{43}{35 x^{7}}\right) t^{7}+\left(\frac{1}{15 x^{2}}-\frac{1019}{720 x^{3}}+\frac{2}{15 x^{4}}+\frac{5}{2 x^{5}}\right) t^{6}+ \\
& \left(\frac{1}{120 x}+\frac{13}{120 x^{2}}-\frac{73}{60 x^{3}}\right) t^{5}+\frac{1}{24 x} t^{4}
\end{aligned}
$$

etc ..., and further terms can easily be calculated using a symbolic computation package.

\subsection{Numerical analysis}

Here, we compare the exact solution obtained by the standard Adomian decomposition method. We take

$U_{N}(x, t)=\sum_{n=0}^{N} u_{n}(x, t)$

$\begin{array}{cccccc}(x, t) & U_{0} & U_{1} & U_{3} & U_{4} & U_{\text {exact }} \\ (0.3,0.1) & 2.5556 & 2.5416 & 2.5009 & 2.5007 & 2.5 \\ (0.4,0.1) & 2.125 & 2.0121 & 2.0001 & 2.0001 & 2.0 \\ (0.5,0.1) & 1.8 & 1.6694 & 1.6667 & 1.6666 & 1.6667\end{array}$

\section{Conclusion}

In this paper, we showed through this example that the modified Adomian decomposition method can be used to solve the wave equation and give us the exact, on the other hand, the standard Adomian decomposition method gives the approached solution, we remark too that the approached solution rapidly converges to the exact solution.

\section{References}

[1] K. ABBAOUI, Les fondements de la méthode décompositionnelle d'Adomian et application à la résolution de problèmes issus de la biologie et de la médecine. Thèse de doctorat de l'Université Paris VI. Octobre 1995.

[2] K. ABBAOUI and Y. CHERRUAULT, Convergence of Adomian method applied to differential equations. Math. Comput. Modelling (28,5), pp.103-109.

[3] K. ABBAOUI and Y. CHERRUAULT, Convergence of Adomian method applied to non linear equations. Math. Comput. Modelling (20,9), pp60-73, 1994.

[4] K. ABBAOUI and Y. CHERRUAULT, The decomposition method applied to the Cauchy problem. Kybernetes, $(28,1)$, pp68-74, 1999.

[5] N. NGARASTA, B. SOME, K.ABBAOUI and Y. CHERRUAULT, New numerical study of Adomian method applied to a diffusion model. Kybernetes, Vol.31, No1, pp61-75, 2002. E.M. DE JAGER.

[6] Gabriel BISSANGA, Application of Adomian decomposition method to solving the Duffing equation. Comparison with perturbation method. Proceedings of the Fourth International Workshop on Contemporary Problems in Mathematical Physics, Cotonou Benin, nov. 2005.

[7] Gabriel BISSANGA. A-K NSEMI. Application of Adomian decomposition method to solving the Van der Pol equation and comparison with the regular perturbation method. Proceedings of the Five international workshop on contemporary problems in mathematical physics, Cotonou-Benin, eds. J. Govaerts, M.N. Hounkounou (International Chair in Mathematical Physics and Applications, ICMP-UNESCO Chair, University of Abomey-Calavy, 072 BP 50 Cotonou, Republic of Benin, December 2008).

[8] Pierre BAKI-TANGOU, Gabriel BISSANGA. Application of Adomian Decomposition method to solving the Duffing-Van der Pol equation. Communications in Mathematical Analysis. Vol. 4, No. pp.30-40(2008).

[9] D. Lesnic, A computational algebraic investigation of the decomposition method for time-dependent problems. Applied Mathematics and Computation 119 (2001) 197-206.

[10] D. Lesnic, Blow-up solutions obtained using the decomposition method. Chaos, Solutions 28 (2006) 776-787.

[11] Chengri Jin, Mingzhu Liu. A new modification of Adomian decomposition method for solving a kind of evolution equation. Applied Mathematics and Computation 169 (2005) 953-962. 